\title{
DESCENT SETS FOR SYMPLECTIC GROUPS
}

\author{
MARTIN RUBEY, BRUCE E. SAGAN, AND BRUCE W. WESTBURY
}

\begin{abstract}
The descent set of an oscillating (or up-down) tableau is introduced. This descent set plays the same role in the representation theory of the symplectic groups as the descent set of a standard tableau plays in the representation theory of the general linear groups. In particular, we show that the descent set is preserved by Sundaram's correspondence. This gives a direct combinatorial interpretation of the branching rules for tensor products of the defining representation of the symplectic groups; equivalently, for the Frobenius character of the action of a symmetric group on an isotypic subspace in a tensor power of the defining representation of a symplectic group.
\end{abstract}

\section{INTRODUCTION}

In this paper we propose a new combinatorial approach to an open problem in representation theory and solve one particular case. The problem can be formulated in very general terms: let $G$ be a connected reductive complex algebraic group and let $V$ be a finite dimensional rational representation of $G$. Then, for $r \geq 0$, the group $G$ acts diagonally on the tensor power $\otimes^{r} V$; moreover the symmetric group, $\mathfrak{S}_{r}$, acts by permuting tensor coordinates. Since these two actions commute we have an action of $G \times \mathfrak{S}_{r}$ on $\otimes^{r} V$. This representation is completely reducible. The problem is then to describe the decomposition of $\otimes^{r} V$ into irreducible components. In particular one would like to determine the characters of the isotypic components as representations of $\mathfrak{S}_{r}$.

There are two examples we consider: the defining representations of $G=\mathrm{GL}(n)$, a general linear group, and $G=\operatorname{Sp}(2 n)$, a symplectic group. The results for the general linear groups are well known, although not from the point of view taken here, while the results for the symplectic groups are new.

Our approach to this problem builds on the combinatorial theory of crystal graphs. We introduce the notion of descent set of a highest weight vertex in a tensor power of a crystal graph. When $V$ is the defining representation of a general linear group, the usual descent set of a word is a descent set in this sense. We then exhibit a descent set for tensor products of the defining representation of a symplectic group. In particular, our main result, Theorem 4.2 below, is obtained

Date: November 4, 2018. 
by showing that the Sundaram correspondence for oscillating tableaux preserves descent sets.

The rest of this article is structured as follows. In the next section we will review our setup in the familiar case of the general linear group. Section 3 then considers any complex reductive algebraic group $G$ and contains our new definition of a descent function in this general setting. Section 4 specializes again to the symplectic case and Section 5 gives the necessary background about the Sundaram and Berele correspondences. The following section gives the proof of our main result by showing that Sundaram's bijection preserves descent sets. In the last section, we explore Roby's description of the Sundaram map using growth diagrams.

\section{Robinson-SCHEnsted And THE GENERAL LineAR GROUPS}

In this section we review some properties of the Robinson-Schensted correspondence and its connection with Schur-Weyl duality. For a textbook treatment we refer to the books of Sagan [10, Chapter 3] or Stanley [12, Chapter 7]. Let $V$ be the defining representation of $\operatorname{GL}(n)$. Then the result due to Schur is that the decomposition of $\otimes^{r} V$ as a representation of $\mathrm{GL}(n) \times \mathfrak{S}_{r}$ is given by

$$
\otimes^{r} V \cong \bigoplus_{\substack{\mu \in P(r) \\ \ell(\mu) \leq n}} V(\mu) \otimes S(\mu)
$$

where $V(\mu)$ is an irreducible representation of $\operatorname{GL}(n)$ and $S(\mu)$ is an irreducible representation of $\mathfrak{S}_{r}$. In the sum, $P(r)$ represents the set of partitions of $r$, and $\ell(\mu)$ is the number of parts of the partition $\mu$.

The Robinson-Schensted correspondence is a bijection

$$
\mathrm{RS}:\{1, \ldots, n\}^{r} \rightarrow \coprod_{\substack{\mu \in P(r) \\ \ell(\mu) \leq n}} \operatorname{SSYT}(\mu, n) \times \operatorname{SYT}(\mu),
$$

where $\operatorname{SSYT}(\mu, n)$ is the set of semistandard Young tableaux of shape $\mu$ with entries less than or equal to $n$, and $\operatorname{SYT}(\mu)$ is the set of standard Young tableaux of shape $\mu$. When $\operatorname{RS}(w)=(P, Q)$, we call $P$ the insertion and $Q$ the recording tableau of $w$.

One relationship between equations (11) and (2) is that the character of $V(\mu)$ is given by the Schur polynomial associated to $\mu$,

$$
s_{\mu}\left(x_{1}, \ldots, x_{n}\right)=\sum_{T \in \operatorname{SSYT}(\mu, n)} \mathbf{x}^{T} .
$$

so, in particular, $V(\mu)$ has a basis indexed by $\operatorname{SSYT}(\mu, n)$. Also, there is a basis of $S(\mu)$ indexed by $\operatorname{SYT}(\mu)$. Furthermore, the RobinsonSchensted correspondence is weight preserving, so its existence constitutes a combinatorial proof of the character identity implied by equation (11). 
Associated to any representation $\rho: \mathfrak{S}_{r} \rightarrow \mathrm{GL}(U)$ is its Frobenius character

$$
\operatorname{ch} \rho=\frac{1}{r !} \sum_{\pi \in \mathfrak{S}_{r}} \operatorname{Tr} \rho(\pi) p_{\lambda(\pi)},
$$

where $\operatorname{Tr}$ denotes the trace, the partition $\lambda(\pi)$ is the cycle type of the permutation $\pi$ and $p_{\lambda(\pi)}$ denotes the associated power sum symmetric function. By abuse of notation we will write $\operatorname{ch} U$ instead of $\operatorname{ch} \rho$. In particular, we have $\operatorname{ch} S(\mu)=s_{\mu}$, where $s_{\mu}$ is the Schur function associated to $\mu$.

To establish the connection with descent sets, we express the Frobenius character of the isotypic component of type $\mu$ in equation (11) in terms of the fundamental quasi-symmetric functions (see [12, Theorem 7.19.7]):

$$
\operatorname{ch} S(\mu)=s_{\mu}=\sum_{Q \in \operatorname{SYT}(\mu)} F_{\operatorname{Des}(Q)}
$$

where, for any subset $D \subseteq\{1,2, \ldots, r-1\}$,

$$
F_{D}=\sum_{\substack{i_{1} \leq i_{2} \leq \cdots \leq i_{r} \\ j \in D \Longrightarrow i_{j}<i_{j+1}}} x_{i_{1}} x_{i_{2}} \ldots x_{i_{r}} .
$$

One of the starting points of this project was the remarkable fact, due to Schützenberger [11, Remarque 2], that the usual descent set of a word equals the descent set of its recording tableau under the RobinsonSchensted correspondence. Moreover, the correspondence restricts to a bijection between reverse lattice permutations (also known as reverse Yamanouchi words) of weight $\mu$ and the set $\operatorname{SYT}(\mu)$.

Thus, we can alternatively describe the Frobenius character as

$$
\operatorname{ch} S(\mu)=\sum_{w} F_{\operatorname{Des}(w)},
$$

where the summation is over all reverse lattice permutations of weight $\mu$. We provide symplectic analogues of equations (44) and (5) in Theorems 4.9 and 4.2 , respectively.

\section{The General SETting}

Let $V$ be a finite dimensional rational representation of a complex reductive algebraic group $G$. Let $\Lambda$ be the set of isomorphism classes of irreducible rational representations of $G$ and let $V(\mu)$ be the representation corresponding to $\mu \in \Lambda$. For example, when $G$ is the general linear group $\mathrm{GL}(n)$ we can identify $\Lambda$ with the set of weakly decreasing sequences of integers of length $n$. Also, when $G$ is the symplectic group $\operatorname{Sp}(2 n)$ we can identify $\Lambda$ with the set of partitions with at most $n$ parts. In both cases, the trivial representation corresponds to the empty partition and the defining representation corresponds to the partition 1 . 
For the symmetric group, $\mathfrak{S}_{r}$, we identify the isomorphism classes of its irreducible representations with the set of partitions of $r, P(r)$. We denote the representation corresponding to $\lambda \in P(r)$ by $S(\lambda)$.

For each $r \geq 0$, the tensor power $\otimes^{r} V$ is a rational representation of $G \times \mathfrak{S}_{r}$, where $G$ acts diagonally and $\mathfrak{S}_{r}$ acts by permuting tensor coordinates. This representation is completely reducible. Decomposing it as a representation of $G$ we obtain the following analogue of equation (1)

$$
\otimes^{r} V \cong \bigoplus_{\mu \in \Lambda} V(\mu) \otimes U(r, \mu) .
$$

The isotypic space $U(r, \mu)$ inherits the action of $\mathfrak{S}_{r}$ and therefore decomposes as

$$
U(r, \mu) \cong \bigoplus_{\lambda \in P(r)} A(\lambda, \mu) \otimes S(\lambda) .
$$

Thus, the Frobenius character of $U(r, \mu)$ is

$$
\operatorname{ch} U(r, \mu)=\sum_{\lambda \in P(r)} a(\lambda, \mu) s_{\lambda},
$$

where $a(\lambda, \mu)=\operatorname{dim} A(\lambda, \mu)$.

When $V$ is the defining representation of $\mathrm{GL}(n)$ the coefficient $a(\lambda, \mu)$ equals 1 for $\lambda=\mu$ and vanishes otherwise. Thus, in this case the Frobenius character is simply $s_{\mu}$. For the defining representation of the symplectic group $\operatorname{Sp}(2 n)$ the coefficients $a(\lambda, \mu)$ were determined by Sundaram [13] and Tokuyama [16]. In general it is a difficult problem to determine these characters explicitly.

We would like to advertise a new approach to this problem, using descent sets. It appears that the proper setting for this approach is the combinatorial theory of crystal graphs. This theory, introduced by Kashiwara, is an off-shoot of the representation theory of DrinfeldJimbo quantised enveloping algebras. For a textbook treatment we refer to the book by Hong and Kang [5].

For each rational representation $V$ of a connected reductive algebraic group there is a crystal graph. The vector space $V$ is replaced by a set of cardinality $\operatorname{dim}(V)$. The raising and lowering operators, which are certain linear operators on $V$, are replaced by partial functions on the set. It is common practice to represent these partial functions by directed graphs. Each arc is coloured according to the application of the Kashiwara operator it represents.

For example, the crystal graph corresponding to the defining representation of $\mathrm{GL}(n)$ is

$$
1 \stackrel{1}{\longrightarrow} 2 \stackrel{2}{\longrightarrow} \cdots \stackrel{n-2}{\longrightarrow} n-1 \stackrel{n-1}{\longrightarrow} n .
$$


while the crystal graph corresponding to the defining representation of $\operatorname{Sp}(2 n)$ is

$$
1 \stackrel{1}{\longrightarrow} 2 \stackrel{2}{\longrightarrow} \cdots \stackrel{n-1}{\longrightarrow} n \stackrel{n}{\longrightarrow}-n \stackrel{n-1}{\longrightarrow}-(n-1) \stackrel{n-2}{\longrightarrow} \cdots \stackrel{1}{\longrightarrow}-1 .
$$

Each vertex of the crystal has a weight and the sum of these weights is the character of the representation, e.g., equation (3) for the general linear group. Isomorphic representations correspond to crystal graphs that are isomorphic as coloured digraphs and the representation is indecomposable if and only if the graph is connected.

A vertex in a crystal graph with no in-coming arcs is a highest weight vertex. Each connected component contains a unique highest weight vertex. The weight of this vertex is the weight of the representation it corresponds to. Thus, the components of a crystal graph are in bijection with the set of highest weight vertices.

There is a (relatively) simple way to construct the crystal graph of a tensor product of two representations given their individual crystal graphs. In particular, the highest weight vertices of the crystal corresponding to $\otimes^{r} V$ can be regarded as words of length $r$ with letters being vertices of the crystal corresponding to $V$.

For example, when $V$ is the defining representation of $\operatorname{GL}(n)$, the highest weight words can be identified with reverse Yamanouchi words, see Definition 5.1. When $V$ is the defining representation of $\operatorname{Sp}(2 n)$ the highest weight words correspond to $n$-symplectic oscillating tableaux, see Definition 4.3 .

Thus we obtain a combinatorial interpretation of equation (6) which is a far-reaching generalisation of the Robinson-Schensted correspondence. Explicit insertion schemes in analogy to the classical correspondence were found corresponding to the defining representation for the symplectic groups $\mathrm{Sp}(2 n)$ as well as for the odd and even orthogonal groups, see [7].

An important feature of these insertion schemes is that they can be understood as isomorphisms of crystals. In the example of the defining representation of GL $(n)$, the Robinson-Schensted correspondence puts the highest weight words in bijection with standard tableaux. Moreover, the words in each component of the crystal have the same recording tableau, and two words have the same insertion tableau if and only if they occur in the same position of two isomorphic components of the crystal graph.

Let us remark that there are other insertion schemes for the classical groups, for example by Berele [1] for the symplectic groups, Okada [8] for the even orthogonal groups and Sundaram [14] for the odd orthogonal groups. However, these are not isomorphisms of crystals.

We can now state the fundamental property we require for a descent set in the general sense. 
Definition 3.1. Suppose that the function Des assigns to each highest weight vertex of the crystal graph corresponding to $\otimes^{r} V$ a subset of $\{1,2, \ldots, r-1\}$. Then Des is a descent function if it satisfies

$$
\operatorname{ch} U(r, \mu)=\sum_{w} F_{\operatorname{Des}(w)}
$$

where the sum is over all highest weight vertices of weight $\mu$.

Thus, by equation (5), the usual descent set of a word is a descent set in the sense of this definition for the defining representation of $\mathrm{GL}(n)$. We note that in terms of the crystal graph (9) above, a highest weight vertex $w_{1} w_{2} \ldots w_{r}$ has a descent at position $k$ if and only if there is a (nontrivial) directed path from $w_{k+1}$ to $w_{k}$ in the crystal graph.

In this article we exhibit a descent function with a similar description for the symplectic groups, see Definition 4.1.

\section{Oscillating tableaux And Descents}

In the case of the defining representation of the symplectic group $\operatorname{Sp}(2 n)$ the vertices of the crystal graph corresponding to $\otimes^{r} V$ are words $w=w_{1} w_{2} \ldots w_{r}$ in $\{ \pm 1, \ldots, \pm n\}^{r}$. The weight of a vertex is the tuple $\operatorname{wt}(w)=\left(\mu_{1}, \ldots, \mu_{n}\right)$, where $\mu_{i}$ is the number of letters $i$ minus the number of letters $-i$ in $w$. The vertex is a highest weight vertex if for any $k \leq r$, the weight of $w_{1} \ldots w_{k}$ is a partition, i.e., $\mu_{1} \geq \mu_{2} \cdots \geq$ $\mu_{n} \geq 0$.

Definition 4.1. A highest weight vertex $w_{1} w_{2} \ldots w_{r}$ in the crystal graph corresponding to $\otimes^{r} V$ has a descent at position $k$ if there is a (nontrivial) directed path from $w_{k}$ to $w_{k+1}$ in the crystal graph (10).

The descent set of $w$ is

$$
\operatorname{Des}(w)=\{k \mid k \text { is a descent of } w\} .
$$

Note that the direction of the path for these descents is opposite from the one used for $\mathrm{GL}(n)$.

We can now state our main result.

Theorem 4.2. Let $V$ be the defining representation of the symplectic group $\operatorname{Sp}(2 n)$. Then the Frobenius character of the isotypic component, $U(r, \mu)$, of $\otimes^{r} V$ is

$$
\operatorname{ch} U(r, \mu)=\sum_{w} F_{\operatorname{Des}(w)},
$$

where the sum is over all highest weight vertices of weight $\mu$ in the corresponding crystal graph.

To prove this theorem we will first rephrase it in terms of $n$-symplectic oscillating tableaux, also known as up-down-tableaux, which are in bijection with the highest weight vertices in the crystal graph corresponding to $\otimes^{r} V$. 
Definition 4.3. An oscillating tableau of length $r$ and (final) shape $\mu$ is a sequence of partitions

$$
\left(\emptyset=\mu^{0}, \mu^{1}, \ldots, \mu^{r}=\mu\right)
$$

such that the Ferrers diagrams of two consecutive partitions differ by exactly one box. We view all Ferrers diagrams in English notation.

The $k$-th step, going from $\mu^{k-1}$ to $\mu^{k}$, is an expansion if a box is added and a contraction if a box is deleted. We will refer to the box that is added or deleted in the $k$-th step as $b_{k}$.

The oscillating tableau $\mathcal{T}=\left(\mu^{0}, \mu^{1}, \ldots, \mu^{r}\right)$ is $n$-symplectic if every partition $\mu^{i}$ has at most $n$ non-zero parts.

The next result follows immediately from the definitions above.

Proposition 4.4. The following is a bijection between $n$-symplectic oscillating tableaux of length $r$ and highest weight vertices $w$ in the crystal graph corresponding to $\otimes^{r} V$ :

- given a highest weight vertex $w_{1} w_{2} \ldots w_{r}$, the oscillating tableau is the sequence of weights of its initial factors

$$
\left(\operatorname{wt}\left(w_{1}\right), \operatorname{wt}\left(w_{1} w_{2}\right), \operatorname{wt}\left(w_{1} w_{2} w_{3}\right), \ldots, \operatorname{wt}\left(w_{1} w_{2} \ldots w_{r}\right)\right)
$$

- for an oscillating tableau $\mathcal{T}$ the corresponding word $w_{1} w_{2} \ldots w_{r}$ is given by $w_{k}= \pm i$, where $i$ is the row of $b_{k}$ and one uses plus or minus if $b_{k}$ is added or deleted, respectively.

Note that in general, the oscillating tableau obtained via Berele's correspondence (see Section 5) from a word $w_{1} w_{2} \ldots w_{r}$ is different from the oscillating tableau given by the bijection above.

Example 4.5. The 1-symplectic oscillating tableaux of length three are

$$
(\emptyset, 1,2,3), \quad(\emptyset, 1,2,1), \quad \text { and } \quad(\emptyset, 1, \emptyset, 1) .
$$

In terms of Ferrers diagrams, these are

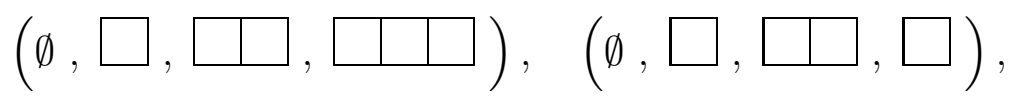

and

$$
(\emptyset, \square, \emptyset, \square)
$$

respectively. The corresponding words are

$$
111, \quad 11-1 \text {, and 1-11. }
$$

As a running example, we will use the oscillating tableau

$$
\mathcal{T}=(\emptyset, 1,11,21,2,1,2,21,211,21)
$$

which has length 9 and shape 21. It is 3-symplectic (since no partition has four parts) but it is not 2-symplectic (since there is a partition with three parts). The corresponding word is

$$
w=121-2-1123-3
$$


with descent set

$$
\operatorname{Des}(w)=\{1,3,4,6,7,8\} .
$$

We will now define the descent set of an oscillating tableau in such a way that a tableau $\mathcal{T}$ and its word $w$ will always have the same descent set.

Definition 4.6. An oscillating tableau $\mathcal{T}$ has a descent at position $k$ if

- step $k$ is an expansion and step $k+1$ is a contraction, or

- steps $k$ and $k+1$ are both expansions and $b_{k}$ is in a row strictly above $b_{k+1}$, or

- steps $k$ and $k+1$ are both contractions and $b_{k}$ is in a row strictly below $b_{k+1}$.

The descent set of $\mathcal{T}$ is

$$
\operatorname{Des}(\mathcal{T})=\{k \mid k \text { is a descent of } \mathcal{T}\} .
$$

Example 4.7. The descent set of the oscillating tableau $\mathcal{T}$ from Example 4.5 is

$$
\operatorname{Des}(\mathcal{T})=\{1,3,4,6,7,8\} .
$$

which is the same as the descent set in (11) as predicted.

The fact that these two descent sets always coincide is easy to prove directly from the definitions, so we omit the proof and just formally state the result.

Proposition 4.8. The descent set of an n-symplectic oscillating tableau of length $r$ coincides with the descent set of the corresponding highest weight vertex of the crystal graph of $\otimes^{r} V$.

Thus it suffices to prove the following variant of Theorem 4.2.

Theorem 4.9. Let $V$ be the defining representation of the symplectic group $\operatorname{Sp}(2 n)$. Then the Frobenius character of the isotypic component, $U(r, \mu)$, of $\otimes^{r} V$ is

$$
\operatorname{ch} U(r, \mu)=\sum_{\mathcal{T}} F_{\operatorname{Des}(\mathcal{T})},
$$

where the sum is over all n-symplectic oscillating tableaux of length $r$ and shape $\mu$.

Let us motivate Definition 4.6 in a second way. Note that a standard Young tableau $T$ can be regarded as an oscillating tableau $\mathcal{T}$ where every step is an expansion and the box containing $k$ in $T$ is added during the $k$-th step in $\mathcal{T}$. In this case

$$
\operatorname{Des}(\mathcal{T})=\operatorname{Des}(T)
$$

where a descent of a standard Young tableau $T$ is an integer $k$ such that $k+1$ appears in a lower row than $k$ of $T$. So the definition of 
descents for oscillating tableaux is an extension of the usual one for standard tableaux.

In Sundaram's correspondence, an arbitrary oscillating tableau $\mathcal{T}$ is first transformed into a fixed-point-free involution $\iota$ on a subset $A$ of the positive integers and a partial Young tableau $T$, that is, a filling of a Ferrers shape with all entries distinct and increasing in rows and columns. There are natural notions of descents for these objects which extend those for permutations in $\mathfrak{S}_{r}$ and standard Young tableaux.

Definition 4.10. Let $A$ be a set of positive integers. The descent set of a bijection $\iota: A \rightarrow A$ is

$$
\operatorname{Des}(\iota)=\{k: k, k+1 \in A, \iota(k)>\iota(k+1)\} .
$$

For a partial Young tableau $T$ whose set of entries is $A$, the descent set is

$$
\operatorname{Des}(T)=\{k: k, k+1 \in A, k+1 \text { is in a row below } k\} .
$$

The definition of the descent set for an oscillating tableau is constructed so that it contains the union of the descent sets of the associated partial tableau and involution under Sundaram's bijection.

\section{The Correspondences of Berele and Sundaram}

One of our main tools for proving Theorem 4.9 will be a bijection, Sun, due to Sundaram [13, 15]. Berele [1] constructed a bijection which is a combinatorial counterpart of the isomrphism in equation (6) when $V$ is the defining representation of $\operatorname{Sp}(2 n)$ In combination with Berele's correspondence, Sundaram's bijection can be regarded in its turn as a combinatorial counterpart of the isomorphism in equation (7). In this section we define the objects involved; the bijection Sun itself will be described in detail in the next section.

Definition 5.1. Let $u$ be a word with letters in the positive integers. Then $u$ is a Yamanouchi word (or lattice permutation) if, in each initial factor $u_{1} u_{2} \ldots u_{k}$, there are at least as many occurrences of $i$ as there are of $i+1$ for all $i \geq 1$. The weight $\beta$ of a lattice permutation $u$ is the partition $\beta=\left(\beta_{1} \geq \beta_{2} \geq \cdots\right)$, where $\beta_{i}$ is the number of occurrences of the letter $i$ in $u$.

For a skew semistandard Young tableau $S$ the reading word, $w(S)$, is the word obtained by concatenating the rows from bottom to top. It has the nice property that applying the Robinson-Schensted map to $w(S)$ one recovers $S$ as the insertion tableau. We will need the reverse reading word, $w^{\text {rev }}(S)$, which is obtained by reading $w(S)$ backwards.

A skew semistandard Young tableau $S$ of shape $\lambda / \mu$ is called an $n$-symplectic Littlewood-Richardson tableau of weight $\beta$ if

- its reverse reading word is a lattice permutation of weight $\beta$, where $\beta$ is a partition with all columns having even length, and 
- entries in row $n+i+1$ of $S$ are greater than or equal to $2 i+2$ for $i \geq 0$.

We will denote the number of $n$-symplectic Littlewood-Richardson tableaux of shape $\lambda / \mu$ and weight $\beta$ by $c_{\mu, \beta}^{\lambda}(n)$.

For $\ell(\lambda) \leq n+1$ we have that the number $c_{\mu, \beta}^{\lambda}(n)$ is the usual Littlewood-Richardson coefficient whenever $\beta$ is a partition with all columns having even length. This is trivial for $\ell(\lambda) \leq n$, and follows from the correspondence Sun described below for $\ell(\lambda)=n+1$.

Note that we are only interested in the case when the length of $\mu$ is at most $n$. In this case the restriction on the size of the entries is equivalent to the condition given by Sundaram that $2 i+1$ appears no lower than row $n+i$ for $i \geq 0$. As an example, when $\mu=(1)$ there is a single 1-symplectic Littlewood-Richardson tableau of weight $\beta=(1,1)$, namely

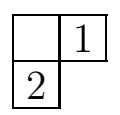

We alert the reader that there is a typographical error in both 13 , Definition 9.5] and [15, Definition 3.9], where the range of indices is stated as $1 \leq i \leq \frac{1}{2} \ell(\beta)$. With this definition,

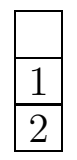

would also be 1 -symplectic, since $\beta=(1,1)$ and the only relevant value for $i$ would be 1 , giving $2 i+1=3$, which does not appear at all in the tableau. However, there are two 1-symplectic oscillating tableaux of length 3 and shape (1), and there are two standard Young tableaux of shape $(2,1)$. Thus, if the tableau above were 1 -symplectic, Theorem 5.2 below would fail.

We now have all the definitions in place to explain the domain and range of the correspondence Sun.

Theorem 5.2 ([13, Theorem 9.4]). The map Sun described below is a bijection between $n$-symplectic oscillating tableaux of length $r$ and shape $\mu$ and pairs $(Q, S)$, where

- $Q$ is a standard tableau of shape $\lambda$, with $|\lambda|=r$, and

- $S$ is an n-symplectic Littlewood-Richardson tableau of shape $\lambda / \mu$ and weight $\beta$, where $\beta$ has even columns and $|\beta|=r-|\mu|$.

For completeness, let us point out the relation between Sundaram's bijection and Berele's correspondence. This correspondence involves the following objects due to King [6], indexing the irreducible representations of the symplectic group $\operatorname{Sp}(2 n)$. 
Definition 5.3. An $n$-symplectic semistandard tableau of shape $\mu$ is a filling of $\mu$ with letters from $1<-1<2<-2<\cdots<n<-n$ such that

- entries in rows are weakly increasing,

- entries in columns are strictly increasing, and

- the entries in row $i$ are greater than or equal to $i$, in the above ordering.

Denoting the set of $n$-symplectic oscillating tableaux of length $r$ and final shape $\mu$ by $\operatorname{Osc}(r, n, \mu)$ and the set of $n$-symplectic semistandard tableaux of shape $\mu$ by $K(\mu, n)$, Berele's correspondence is a bijection

$$
\text { Ber : }\{ \pm 1, \ldots, \pm n\}^{r} \rightarrow \bigcup_{\ell(\mu) \leq n} K(\mu, n) \times \operatorname{Osc}(r, n, \mu) \text {. }
$$

Analogous to equation (3), the character of the representation $V(\mu)$ of the symplectic group $\operatorname{Sp}(2 n)$ is

$$
s p_{\mu}\left(x_{1}^{ \pm 1}, \ldots, x_{n}^{ \pm 1}\right)=\sum_{T \in K(\mu, n)} \mathbf{x}^{T} .
$$

Now consider an $n$-symplectic oscillating tableau as a word in the ordered alphabet $1<-1<2<-2<\cdots<n<-n$, as described just after Definition 4.3. We can then apply the Robinson-Schensted correspondence to obtain a semistandard Young tableau $P_{\mathrm{RS}}$ in this alphabet and an (ordinary) standard Young tableau $Q_{\mathrm{RS}}$. Alternatively, we can compose Berele's correspondence with Sundaram's bijection to obtain a triple $\left(P_{\mathrm{Ber}}, Q_{\mathrm{Sun}}, S_{\mathrm{Sun}}\right)$. It then turns out that $Q_{\mathrm{RS}}=Q_{\mathrm{Sun}}$. This implies that for each standard Young tableau $Q_{\text {Sun }}$ we have a correspondence $P_{\mathrm{RS}} \mapsto\left(P_{\mathrm{Ber}}, S_{\mathrm{Sun}}\right)$. Moreover, this correspondence is independent of the choice of $Q_{\text {Sun }}$. One can then prove the following theorem.

Theorem 5.4 ([13, Theorem 12.1]). If $V$ is the defining representation of $\operatorname{Sp}(2 n)$ then the coefficient $a(\lambda, \mu)$ is given by

$$
a(\lambda, \mu)=\sum_{\beta} c_{\mu, \beta}^{\lambda}(n)
$$

where the sum is over the partitions $\beta$ of $|\lambda|-|\mu|$ having only columns of even length.

Let us point out a corollary, which settles a conjecture from [17].

Corollary 5.5. If $V$ is the defining representation of $\operatorname{Sp}(2 n)$ then the Frobenius character of the isotypic component of $\otimes^{r} V$ of weight 0 is given by

$$
\operatorname{ch} U(r, \emptyset)=\sum_{\beta} s_{\beta},
$$

where the sum is over the partitions $\beta$ of $r$ such that if $c$ is any column length of $\beta$ then $c$ is even and $c \leq 2 n$. 
Proof. Comparing equation (8) with the previous Theorem, it suffices to show that $c_{\emptyset, \beta}^{\lambda}$ is nonzero only when $\lambda=\beta$ is a partition of the type described in the corollary, and in this case there is only one corresponding tableau. Suppose $S$ is an $n$-symplectic Littlewood-Richardson tableau of shape $\lambda / \emptyset$ and weight $\beta$. Then $S$ is a semistandard Young tableau of straight shape $\lambda$ whose reverse reading word is a lattice permutation. This implies that all entries in row $j$ of $S$ are equal to $j$. In particular, $\lambda=\beta$ and $\beta$ satifies the conditions of Theorem 5.4. Furthermore, is is required that the entries in row $j=n+i+1$ are at least $2 i+2$ for $i \geq 0$. It follows that $i+1 \leq n$, and therefore $j \leq 2 n$.

\section{Proof of the Main Result}

In light of Sundaram's results, we claim that to prove Theorem 4.9 it suffices to demonstrate the following.

Theorem 6.1. Let $\operatorname{Sun}(\mathcal{T})=(Q, S)$. Then

$$
\operatorname{Des}(\mathcal{T})=\operatorname{Des}(Q) .
$$

Proof of Theorem 4.9. We have

$$
\begin{aligned}
& \sum_{\mathcal{T}} F_{\operatorname{Des}(\mathcal{T})}=\sum_{(Q, S)} F_{\operatorname{Des}(Q)} \quad \text { (by Theorems 5.2 and 6.1) } \\
& =\sum_{|Q|=r} \sum_{\beta} c_{\mu, \beta}^{\operatorname{sh} Q}(n) F_{\operatorname{Des}(Q)} \quad \text { (by Definition 5.1) } \\
& =\sum_{|Q|=r} a(\operatorname{sh}(Q), \mu) F_{\operatorname{Des}(Q)} \quad \text { (by Theorem 5.4) } \\
& =\sum_{\lambda \in P(r)} a(\lambda, \mu) \sum_{Q \in \operatorname{SYT}(\lambda)} F_{\operatorname{Des}(Q)} \\
& =\operatorname{ch} U(r, \mu) \quad \text { (by equations (4) and (8) })
\end{aligned}
$$

which is the desired conclusion.

In order to prove Theorem 6.1, we will need to analyse the bijection Sun in detail. Sundaram described Sun as the composition of several bijections. Specifically, Sun is the composition

$$
\mathcal{T} \stackrel{\operatorname{Sun}_{1}}{\mapsto}(\iota, T) \stackrel{\text { RS }}{\mapsto}(I, T) \stackrel{\operatorname{Sun}_{2}}{\mapsto}(Q, S)
$$

where $\operatorname{Sun}_{1}$ and $\operatorname{Sun}_{2}$ are described below and, as usual, RS denotes the Robinson-Schensted correspondence. We will prove Theorem 6.1 by tracking the effect of these maps on the descent set.

6.1. Sundaram's first bijection. We now describe Sundaram's first bijection which we will denote by $\mathrm{Sun}_{1}$. It maps an oscillating tableau $\mathcal{T}$ to a pair $(\iota, T)$ where $\iota$ is a fixed-point-free involution, $T$ is a partial Young tableau, and the entries of $\iota$ and $T$ are complementary sets. 
Let $\mathcal{T}=\left(\emptyset=\mu^{0}, \mu^{1}, \ldots, \mu^{r}\right)$ be an oscillating tableau. We then construct a sequence of pairs $\left(\iota_{k}, T_{k}\right)$ for $0 \leq k \leq r$ such that $T_{k}$ has shape $\operatorname{sh}\left(T_{k}\right)=\mu^{k}$, and the entries of $\iota_{k}$ and of $T_{k}$ form a set partition of $\{1, \ldots, k\}$.

Both $\iota_{0}$ and $T_{0}$ are empty. For $k>0$ the pair $\left(\iota_{k}, T_{k}\right)$ is constructed from the pair $\left(\iota_{k-1}, T_{k-1}\right)$ and the $k$-th step in the oscillating tableau.

- If the $k$-th step is an expansion then $\iota_{k}=\iota_{k-1}$ and $T_{k}$ is obtained from $T_{k-1}$ by putting $k$ in box $b_{k}$.

- If the $k$-th step is a contraction then take $T_{k-1}$ and bump out (using Robinson-Schensted column deletion) the entry in box $b_{k}$ to get a letter $x$ and the partial Young tableau $T_{k}$. The involution $\iota_{k}$ is $\iota_{k-1}$ with the transposition $(x, k)$ adjoined.

The result of the bijection is the final pair $\left(\iota_{r}, T_{r}\right)$.

Lemma 6.2 (Sundaram [13, Lemma 8.7]). The map $\mathrm{Sun}_{1}$ is a bijection between oscillating tableaux of length $r$ and shape $\mu$ and pairs $(\iota, T)$ where

- $\iota$ is a fixed-point-free involution of a set $A \subseteq\{1, \ldots, r\}$, and

- $T$ is a partial tableau of shape $\mu$ such that its set of entries is $\{1, \ldots, r\} \backslash A$.

In general it seems that, given the pair $(\iota, T)$, there is no straightforward way to determine whether the corresponding oscillating tableau is $n$-symplectic, see [13, Lemma 9.3]. However, when $\mu=\emptyset$ the map $\mathrm{Sun}_{1}$ is in fact a bijection between $n$-symplectic oscillating tableaux and $(n+1)$-nonnesting perfect matchings of $\{1,2, \ldots, r\}$, see [2].

Example 6.3. Starting with the oscillating tableau $\mathcal{T}$ from Example 4.5, we get the following sequence of pairs $\left(T_{k}, i_{k}\right)$, where in the diagram below we only list each pair of the involution once when it is produced by the algorithm.

$$
\begin{aligned}
& \begin{array}{lllllllllll}
k: & 0 & 1 & 2 & 3 & 4 & 5 & 6 & 7 & 8 & 9
\end{array}
\end{aligned}
$$

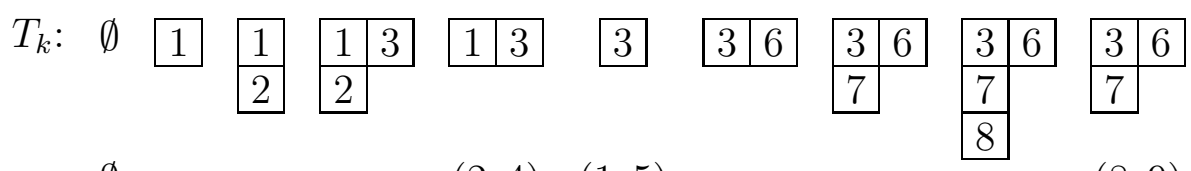

$$
\begin{aligned}
& \iota_{k}: \emptyset \quad(2,4) \quad(1,5)
\end{aligned}
$$

So the final output is the involution

$$
\iota=(1,5)(2,4)(8,9)=\begin{array}{llllll}
1 & 2 & 4 & 5 & 8 & 9 \\
5 & 4 & 2 & 1 & 9 & 8
\end{array}
$$

and the partial tableau

$$
T=\begin{array}{|l|l|}
\hline 3 & 6 \\
\hline 7 &
\end{array}
$$

with $\iota \cup T=\{1, \ldots, 9\}$. 
We wish to define the descent set of a pair $(\iota, T)$ in such a way that this map preserves descents. Let us define for any pair $A$ and $B$

$$
\operatorname{Des}(A / B)=\{k: k \in A \text { and } k+1 \in B\} .
$$

and

$$
\operatorname{Des}(\iota, T)=\operatorname{Des}(\iota) \cup \operatorname{Des}(T) \cup \operatorname{Des}(T / \iota),
$$

where we use Definition 4.10 for the descent sets of $\iota$ and T. Thus, in our running example,

$$
\operatorname{Des}(\iota)=\{1,4,8\}, \operatorname{Des}(T)=\{6\}, \operatorname{Des}(T / \iota)=\{3,7\},
$$

so that $\operatorname{Des}(\iota, T)=\{1,3,4,6,7,8\}$, which coincides with $\operatorname{Des}(\mathcal{T})$.

We can now take our first step in proving Theorem 6.1.

Proposition 6.4. Let $\mathcal{T}$ be an oscillating tableau and suppose that $\operatorname{Sun}_{1}(\mathcal{T})=(\iota, T)$. Then

$$
\operatorname{Des}(\mathcal{T})=\operatorname{Des}(\iota, T) .
$$

Proof. We proceed by analysing the effect of two successive steps in the oscillating tableau.

If step $k$ is an expansion and step $k+1$ is a contraction then $k+1 \in \iota$ and $\iota(k+1)<k+1$. Now $k$ either ends up in $T$ or in $\iota$. In the former case, $k \in \operatorname{Des}(T / \iota)$. In the latter case $\iota(k)>k \geq \iota(k+1)$ and $k \in \operatorname{Des}(\iota)$. In both cases this gives a descent of $(\iota, T)$.

If step $k$ is a contraction and step $k+1$ is an expansion then $k \in \iota$ and $\iota(k)<k$. Now either $k+1$ ends up in $T$ or in $\iota$. In the former case $k \in \operatorname{Des}(\iota / T)$ rather than $\operatorname{Des}(T / \iota)$. In the latter case $\iota(k)<k<$ $k+1<\iota(k+1)$. Neither of these cases gives a descent of $(\iota, T)$.

If steps $k$ and $k+1$ are both contractions then $k, k+1 \in \iota$. If $b_{k}$ is strictly below $b_{k+1}$ then, by well-known properties of $\mathrm{RS}$, the element removed when bumping out $b_{k}$ will be in a lower row than the one obtained when bumping out $b_{k+1}$. Thus $\iota(k)>\iota(k+1)$ and $k \in \operatorname{Des}(\iota, T)$ as desired. By a similar argument, if $b_{k}$ is weakly above $b_{k+1}$ then $\iota(k)<\iota(k+1)$ and $k \notin \operatorname{Des}(\iota, T)$.

Now suppose steps $k$ and $k+1$ are both expansions. If $b_{k}$ is in a row strictly above $b_{k+1}$, then any column deletion will keep $k$ in a row strictly above $k+1$. It follows that at the end we have one of three possibilities. The first is that $k, k+1 \in T$ and, as was just observed, we must have $k \in \operatorname{Des}(T)$. If either element is removed, then $k+1$ must be removed first because the row condition forces $k+1$ to always be in a column weakly left of $k$. So at the end we either have $k \in T$ and $k+1 \in \iota$, or we have $\iota(k)>\iota(k+1)$. So in every case $k \in \operatorname{Des}(\iota, T)$. By a similar argument, if $b_{k}$ is in a row weakly below $b_{k+1}$, then $k \notin \operatorname{Des}(\iota, T)$.

This completes the check of all the cases and the proof. 
6.2. Robinson-Schensted. The next step of the map Sun is to apply the Robinson-Schensted correspondence to the fixed-point-free involution $\iota$ to obtain a partial Young tableau $I$ with the same set of entries as $\iota$. Let us first recall the following well-known facts about the RobinsonSchensted map.

Lemma 6.5 ([10, Exercise 22a] and [12, Exercise 7.28a]). Let $\pi$ be a permutation with $\mathrm{RS}(\pi)=(P, Q)$. Then

- $\operatorname{Des}(\pi)=\operatorname{Des}(Q)$, and

- $\pi$ is a fixed-point-free involution if and only if $P=Q$ and all columns of $Q$ have even length.

Combining Proposition 6.4 with this lemma we obtain the following result.

Proposition 6.6. Let $\mathcal{T}$ be an oscillating tableau, let $\operatorname{Sun}_{1}(\mathcal{T})=(\iota, T)$ and let $\operatorname{RS}(\iota)=(I, I)$. Then

$$
\operatorname{Des}(\mathcal{T})=\operatorname{Des}(I) \cup \operatorname{Des}(T) \cup \operatorname{Des}(T / I) .
$$

Example 6.7. The fixed point free involution from Example 6.3 is mapped to the tableau

$$
I=\begin{array}{|l|l|}
\hline 1 & 8 \\
\hline 2 & 9 \\
\hline 4 & \multicolumn{1}{|l}{} \\
\cline { 1 - 1 } 5 &
\end{array}
$$

6.3. Sundaram's second bijection. To finish defining Sun, we need a bijection $\mathrm{Sun}_{2}$ that transforms the pair of partial Young tableaux $(I, T)$ to a pair $(Q, S)$ as in Theorem 5.2 .

Let $Q$ be the standard Young tableau of shape $\lambda$ obtained by columninserting the reverse reading word of the tableau $I$ into the tableau $T$. Also construct a skew semistandard Young tableau $S$ as follows: whenever a letter of $w^{r e v}(I)$ is inserted, record its row index in $I$ in the box which is added. Then $\operatorname{Sun}_{2}(I, T)=(Q, S)$. Sundaram actually defines this map for pairs $(I, T)$ of semistandard Young tableaux. But we will not need this level of generality.

Lemma 6.8 (Sundaram [13, Theorem 8.11, Theorem 9.4]). The map $\mathrm{Sun}_{2}$ is a map from pairs of partial Young tableaux $(I, T)$ of shapes $\beta$ and $\mu$, respectively, such that $I \bullet T=\{1, \ldots, r\}$ to pairs $(Q, S)$ such that

- $Q$ is a standard Young tableau of shape $\lambda \in P(r)$ and

- $S$ is a semistandard tableau of shape $\lambda / \mu$ and weight $\beta$.

Furthermore, $\mathrm{Sun}_{2}$ becomes a bijection if one restricts the domain to those $(I, T)$ which correspond by $\mathrm{Sun}_{1}$ to an $n$-symplectic oscillating tableau, and the range to those $(Q, S)$ where $S$ is an $n$-symplectic Littlewood-Richardson tableau. 
Example 6.9. The tableau $I$ from Example 6.7 has $w^{\text {rev }}(I)=819245$. Column inserting this word into the tableau $T$ from Example 6.3 and recording the row indices yields the pair of tableaux

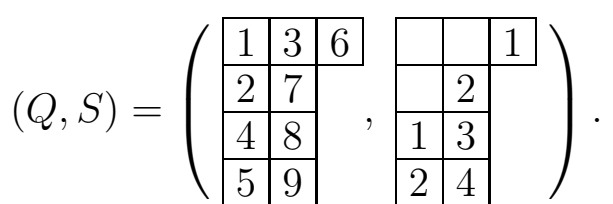

Note that the $\operatorname{Des}(Q)=\{1,3,4,6,7,8\}=\operatorname{Des}(\mathcal{T})$ as desired.

To prove Theorem 6.1 we need two properties of column insertion.

Lemma 6.10. Let $\pi$ be a permutation and suppose that $T$ is obtained by column inserting $\pi$. Then

$$
\operatorname{Des}(T)=\{k: k \text { is left of } k+1 \text { in the 1-line notation for } \pi\} .
$$

Furthermore, column inserting the reverse reading word $w^{\mathrm{rev}}(T)$ of $T$ yields $T$ itself.

Proof of Theorem 6.1. By the first assertion of Lemma 6.10 we have that $Q$ can also be obtained by column-inserting the concatenation of $w^{\mathrm{rev}}(T)$ and $w^{\mathrm{rev}}(I)$. By the second assertion of Lemma 6.10, the descent set of $Q$ equals $\operatorname{Des}(I) \cup \operatorname{Des}(T) \cup \operatorname{Des}(T / I)$, which in turn equals the descent set of $\mathcal{T}$ by Proposition 6.6.

\section{Growth Diagrams and Roby's Description of Sun}

Using Fomin's framework of growth diagrams [3, 4, Roby [9] showed how to obtain the standard tableau $Q$ directly from the oscillating tableau $\mathcal{T}$. However, he omitted the proof that his construction does indeed correspond with that of Sundaram's. We take the opportunity here to review Roby's construction and provide this proof. Using his example Roby also attempted to obtain a direct description of the $n$-symplectic Littlewood-Richardson tableau. However, he made the assumption that a permutation can be partitioned into disjoint increasing subsequences whose lengths are given by the lengths of the rows of the standard Young tableaux associated via the Robinson-Schensted correspondence, which is not true in general.

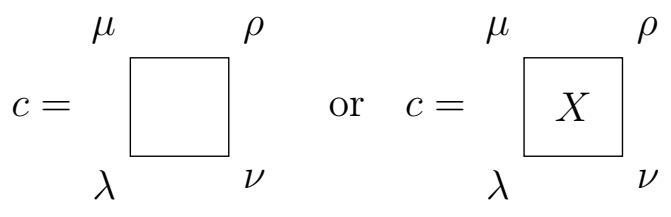

Figure 1. A cell of a growth diagram.

A growth diagram is a rectangular grid sitting inside the first quadrant of a Cartesian coordinate system and composed of cells, $c$ as in 
Figure 1. The four corners of $c$ are labeled with partitions as indicated, and if two partitions are connected by an edge (e.g., $\lambda$ and $\nu$ in Figure 1) then the one closer to the origin either equals or is covered (in Young's lattice) by the one further away. In addition, each cell is either empty or contains a cross, $X$, where we insist that there is at most one cross in every column and every row of the diagram. There are certain local rules according to which the cells are labelled which we explain below. An example for a growth diagram is depicted in Figure 2.

In the following we use the notation $\lambda \lessdot \mu$ to denote that $\lambda$ is covered by $\mu$. Furthermore, for an integer $i$ and a partition $\lambda$ we denote by $\lambda+\epsilon_{i}$ the partition obtained from $\lambda$ by adding one to the $i$ th part, assuming that $\lambda_{i}<\lambda_{i-1}$. Similarly we define $\lambda-\epsilon_{i}$.

The forward rules determine the partition $\rho$ given the other three partitions and whether the cell has a cross or not. They are as follows, organized by the relationship between $\mu$ and $\nu$, and then secondarily by their relationship to $\lambda$.

F1 If $\mu \neq \nu$, then let $\rho=\mu \cup \nu$.

F2 If $\lambda \lessdot \mu=\nu$ then we must have $\mu=\lambda+\epsilon_{i}$ for some $i$, so let $\rho=\mu+\epsilon_{i+1}$.

F3 If $\lambda=\mu=\nu$, then let

$$
\rho= \begin{cases}\lambda & \text { if } c \text { does not contain an } X, \\ \lambda+\epsilon_{1} & \text { if } c \text { contains an } X .\end{cases}
$$

Thus, given the partitions $\lambda, \mu$ and $\nu$ and knowing whether the cell contains a cross or is empty, we can compute the partition $\rho$. An important fact is that this process is invertible in the following sense: given the partitions $\mu, \nu$ and $\rho$ we can compute the partition $\lambda$ as well as the contents of the cell by using the following backward rules.

B1 If $\mu \neq \nu$, then let $\lambda=\mu \cap \nu$.

B2 If $\rho>\mu=\nu$ then we must have $\mu=\rho-\epsilon_{i}$ for some $i$, so let

$$
\lambda= \begin{cases}\mu & \text { if } i=1 \\ \mu-\epsilon_{i-1} & \text { if } i \geq 2 .\end{cases}
$$

where $c$ gets filled with an $X$ in the first case and is left empty in the second.

B3 If $\rho=\mu=\nu$ then let $\lambda=\mu$.

One can visualize these rules as follows. For rules F1 and B1 we have the following possibilities, where in the first diagram $i \neq j$,

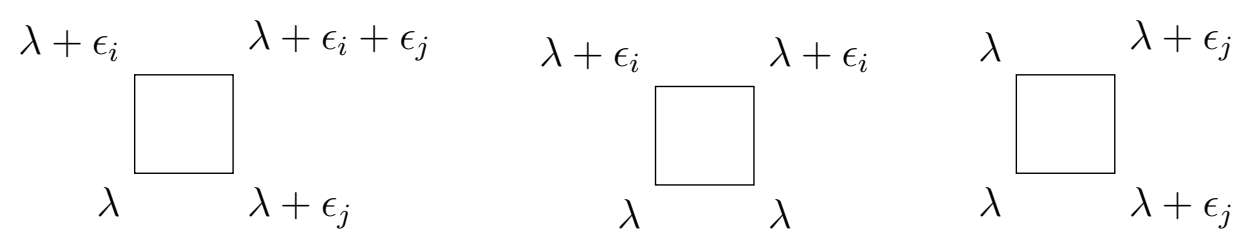


For rules F2-F3 and B2-B3, we can draw the following diagrams
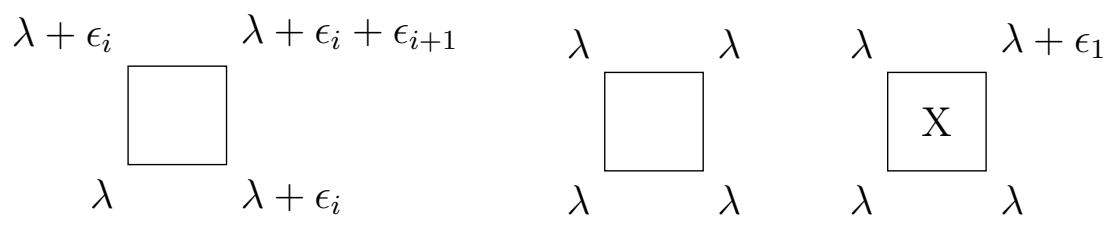

The local rules are, in fact, just another way to perform RobinsonSchensted insertion. To explain this, consider sequences of partitions $\epsilon=\left(\epsilon_{0}, \epsilon_{1}, \ldots, \epsilon_{r}\right)$ where $\epsilon_{0}=\emptyset$ and, for $k>0, \epsilon_{k}$ either equals $\epsilon_{k-1}$ or is obtained from $\epsilon_{k-1}$ by adding a box. Such a sequence $\epsilon$ corresponds to a partial Young tableau $P$ in the same way that an oscillating tableau with all steps expansions corresponds to a standard Young tableau: if $\epsilon_{k}=\epsilon_{k-1}$ then $k$ does not appear in $P$; and if $\epsilon_{k}$ is obtained by adding a box to $\epsilon_{k-1}$ then $k$ appears in $P$ in the box added. Note that if the border of any row in a growth diagram starts with the empty partition, then the sequence of diagrams along this border satisfies the conditions for such an $\epsilon$.

Theorem 7.1 (see [12, Theorem 7.13.5]). Consider a growth diagram consisting of a single row whose cell in column $x$ contains a cross and whose left border is labeled with empty partitions. If the lower and upper borders are identified with partial tableaux $P$ and $P^{\prime}$, respectively, then $P^{\prime}$ is obtained by row inserting $x$ into $P$.

Note that, usually, this theorem is stated with the roles of columns and rows interchanged. However, this is without consequence since the local rules are symmetric in this respect.

We can now explain Roby's description of Sundaram's correspondence. As before, let $\mathcal{T}=\left(\emptyset=\mu_{0}, \mu_{1}, \ldots, \mu_{r}\right)$ be an $n$-symplectic oscillating tableau. We then proceed as follows.

R1 Label the corners of the cells along the main diagonal, i.e., from north-west to south-east, of an $r \times r$ grid with the conjugates of these partitions.

R2 Using the rules B1-B4, determine the partitions labelling the corners of the cells below the main diagonal and which of these cells contain a cross. (Since neighboring partitions on the diagonal will always be distinct, rule B1 will always apply to determine the subdiagonal without needing to know $\rho$.)

R3 Place crosses into those cells above the main diagonal, whose image under reflection about the main diagonal contains a cross.

R4 Using the rules F1-F4, compute the partitions labelling the corners of the cells above the main diagonal.

If we apply this procedure to our running example tableau,

$$
\mathcal{T}=(\emptyset, 1,11,21,2,1,2,21,211,21),
$$




\begin{tabular}{|c|c|c|c|c|c|c|c|c|c|}
\hline & 1 & 2 & 21 & 31 & $X^{41}$ & 411 & 421 & 431 & 441 \\
\hline & 1 & 2 & 21 & $X^{31}$ & 31 & 311 & 321 & 331 & 431 \\
\hline & 1 & 2 & 21 & 21 & 21 & 211 & 221 & 321 & 421 \\
\hline & 1 & $X^{2}$ & 21 & 21 & 21 & 211 & 221 & 321 & 421 \\
\hline & $X^{1}$ & 1 & 11 & 11 & 11 & 111 & 211 & 311 & 411 \\
\hline & $\emptyset$ & $\emptyset$ & 1 & 1 & 1 & 11 & 21 & 31 & 41 \\
\hline & $\emptyset$ & $\emptyset$ & 1 & 1 & 1 & 11 & 21 & 31 & 41 \\
\hline & $\emptyset$ & $\emptyset$ & 1 & 1 & 1 & 11 & 21 & 31 & $X^{41}$ \\
\hline & $\emptyset$ & $\emptyset$ & 1 & 1 & 1 & 11 & 21 & $X^{31}$ & 31 \\
\hline & $\emptyset$ & 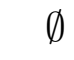 & 1 & 1 & 1 & 11 & 21 & 21 & \\
\hline
\end{tabular}

FiguRE 2. The example growth diagram.

then the corresponding oscillating tableau of conjugate (transposed) partitions is

$$
\mathcal{T}^{t}=(\emptyset, 1,2,21,11,1,11,21,31,21)
$$

and we obtain the growth diagram in Figure 2 ,

Note that the partitions labelling the corners along the left border must all be empty, since $\mu_{0}$, the first partition of the oscillating tableau, is the empty partition. So each row can be associated with a partial Young tableau. Let us single out two particular sequences of partitions: $\left(\emptyset=\kappa_{0}, \kappa_{1}, \ldots, \kappa_{r}\right)$, labelling the corners along the upper border of the growth diagram, and $\left(\emptyset=\tau_{0}, \tau_{1}, \ldots, \tau_{r}\right)$ labelling the corners along the lower border of the growth diagram. In our running example these are

$$
(\emptyset, 1,2,21,31,41,411,421,431,441)
$$

and

$$
(\emptyset, \emptyset, \emptyset, 1,1,1,11,21,21,21)
$$

respectively.

Now let

- $A_{\text {Rob }}$ be the set of column indices of the growth diagram that contain a cross where indices are taken as in a matrix,

- $\iota_{\text {Rob }}$ be the involution of $A_{\text {Rob }}$ that exchanges column and row indices of the cells containing crosses, 
- $T_{\text {Rob }}$ be the partial Young tableau defined by $\left(\emptyset=\tau_{0}^{t}, \ldots, \tau_{r}^{t}\right)$, and

- $Q_{\text {Rob }}$ be the partial Young tableau defined by $\left(\emptyset=\kappa_{0}^{t}, \ldots, \kappa_{r}^{t}\right)$.

In our example, $A_{\text {Rob }}=\{1,2,4,5,8,9\}, \iota_{\text {Rob }}=(1,5)(2,4)(8,9)$,

$$
T_{\text {Rob }}=\begin{array}{|l|l}
\hline 3 & 6 \\
\hline 7 & \text { and } \quad Q_{\text {Rob }}=\begin{array}{l|l|l|}
\hline 1 & 3 & 6 \\
\hline 2 & 7 & \\
\hline 4 & 8 \\
\hline 5 & 9 & \\
\hline
\end{array}
\end{array}
$$

We now compute a second growth diagram. Consider a square grid having crosses in the same places as determined before. Then, label the corners along the lower and the left border of the grid with the empty partition. Finally, using the rules F1-F4 compute the sequence of partitions $\left(\emptyset=\nu_{0}, \nu_{1}, \ldots, \nu_{r}\right)$, labelling the corners along the upper border of the growth diagram. Let $I_{\text {Rob }}$ be the partial Young tableau defined by $\left(\emptyset=\nu_{0}^{t}, \ldots, \nu_{r}^{t}\right)$.

In our example, this sequence is $(\emptyset, 1,2,2,3,4,4,4,41,42)$ with associated tableau

$$
I_{\text {Rob }}=\begin{array}{|l|l|}
\hline 1 & 8 \\
\hline 2 & 9 \\
\hline 4 & \\
\cline { 1 - 1 } 5 &
\end{array} .
$$

Comparing these objects with the ones computed using Sundaram's correspondence, the reader will have anticipated the following theorem.

Theorem 7.2. If

$$
\mathcal{T} \stackrel{\operatorname{Sun}_{1}}{\mapsto}(\iota, T) \stackrel{R S}{\mapsto}(I, T) \stackrel{\operatorname{Sun}_{2}}{\mapsto}(Q, S)
$$

with $\iota$ an involution on $A$, then $A_{\mathrm{Rob}}=A, \iota_{\mathrm{Rob}}=\iota, I_{\mathrm{Rob}}=I, T_{\text {Rob }}=T$ and $Q_{\mathrm{Rob}}=Q$

As mentioned at the beginning of this section it appears that there is no straightforward way to extract the skew Littlewood-Richardson tableau $S$ from the growth diagram.

For the proof the following simple observation will be helpful.

Lemma 7.3. Suppose that the left border of a growth diagram is labelled by empty partitions and let $c$ be a cell labelled with partitions as in Figure 1. Then $\lambda=\mu$ if and only if none of the cells to the left of $c$ and in the same row as contains a cross.

Proof of Theorem [7.2. Let us first show that $A_{\mathrm{Rob}}=A, \iota_{\mathrm{Rob}}=\iota$ and $T_{\text {Rob }}=T$. We will use induction on the length $r$ of the oscillating tableau $\mathcal{T}$. There is nothing to show if $\mathcal{T}$ is empty. Thus, suppose that $r>0$ and that $\mathcal{T}$ with the last step removed is mapped to the pair $\left(\iota^{\prime}, T^{\prime}\right)$ where $\iota^{\prime}$ is an involution on $A^{\prime}$.

If the last step of $\mathcal{T}=\left(\mu_{0}, \ldots, \mu_{r}\right)$ is an expansion, then we claim that the bottom row of the growth diagram does not contain a cross. 
Since $\mu_{r-1} \lessdot \mu_{r}$ the partition $\tau_{r-1}$ is obtained by applying rule B1 and we have $\tau_{r-1}=\mu_{r-1}$. Now the claim follows from the lemma.

We now have that $A_{\text {Rob }}=A^{\prime}$ and $\iota_{\text {Rob }}=\iota^{\prime}=\iota$ in agreement with Sundaram's first correspondence $\operatorname{Sun}_{1}$. Moreover, since we have that $\tau_{r-1}=\mu_{r-1} \lessdot \mu_{r}=\tau_{r}, T_{\text {Rob }}$ is obtained from $T^{\prime}$ by putting $r$ into the cell added by the expansion, which coincides with $T$ as constructed via $\operatorname{Sun}_{1}$.

If the last step of $\mathcal{T}$ is a contraction, again using the lemma, the rule B1 entails that there must be a cross in the bottom row of the growth diagram, say in column $x$. Thus, $\iota_{\text {Rob }}$ is obtained from $\iota^{\prime}$ by adjoining the pair $(x, r)$. It remains to show that $T_{\text {Rob }}$ is obtained by a column deletion of $T^{\prime}$ where $x$ is the element removed at the end of the deletion process. Thus we must show that column inserting $x$ into $T$ yields $T^{\prime}$, or equivalently, that row inserting $x$ into $T^{t}$ yields $\left(T^{\prime}\right)^{t}$. This last statement follows immediately from Theorem 7.1 ,

We now turn to the computation of $Q$ and $Q_{\text {Rob }}$. Using $\operatorname{Sun}_{2}$ the tableau $Q$ is obtained by column inserting the reverse reading word of $I$ into $T$. Equivalently, $Q^{t}$ is obtained by row inserting $w^{\text {rev }}(I)$ into $T^{t}$. Because $w^{r e v}(I)$ is Knuth equivalent to the reversal of the involution $\iota$ in one line notation, Theorem 7.1 shows that the tableau described by the partitions along the upper border of the growth diagram is indeed $Q^{t}$.

The equality of $I$ and $I_{\text {Rob }}$ also follows directly from Theorem 7.1 .

One nice feature of the growth diagram formulation of Sundaram's correspondence is that the descent set can be visualized. Namely, take any partial permutation with insertion tableau $T^{t}$ and construct its growth diagram. On top of this stack the growth diagram for the oscillating tableau so that the two rows corresponding to $T^{t}$ coincide. Then $k$ is a descent in the oscillating tableau if and only if the cross in column $k$ is lower than the cross in column $k+1$. It is in fact possible to prove Theorem 6.1 using this approach.

\section{REFERENCES}

[1] Allan Berele, A Schensted-type correspondence for the symplectic group, J. Combin. Theory Ser. A 43 (1986), no. 2, 320-328. MR 867655 (88b:20027)

[2] William Y. C. Chen, Eva Y. P. Deng, Rosena R. X. Du, Richard P. Stanley, and Catherine H. Yan, Crossings and nestings of matchings and partitions, Transactions of the American Mathematical Society 359 (2007), no. 4, 15551575 (electronic). MR 2272140 (2007i:05015)

[3] Sergey V. Fomin, Duality of graded graphs, Journal of Algebraic Combinatorics 3 (1994), no. 4, 357-404. MR 1293822 (95i:05088)

[4] _ Schensted algorithms for dual graded graphs, Journal of Algebraic Combinatorics 4 (1995), no. 1, 5-45. MR 1314558 (95m:05246)

[5] Jin Hong and Seok-Jin Kang, Introduction to quantum groups and crystal bases, Graduate Studies in Mathematics, vol. 42, American Mathematical Society, Providence, RI, 2002. MR 1881971 (2002m:17012) 
[6] Ron C. King, Weight multiplicities for the classical groups, Group theoretical methods in physics (Fourth Internat. Colloq., Nijmegen, 1975), Springer, Berlin, 1976, pp. 490-499. Lecture Notes in Phys., Vol. 50. MR 0480895 (58 \#1044)

[7] Combinatorics of crystal graphs for the root systems of types $A_{n}, B_{n}, C_{n}, D_{n}$ and $G_{2}$, Combinatorial aspect of integrable systems, MSJ Mem., vol. 17, Math. Soc. Japan, Tokyo, 2007, pp. 11-41. MR 2269126 (2008b:05183)

[8] Soichi Okada, A Robinson-Schensted-type algorithm for $\operatorname{SO}(2 n, \mathbf{C})$, J. Algebra 143 (1991), no. 2, 334-372. MR 1132576 (92k:05133)

[9] Tom Roby, Applications and extensions of Fomin's generalization of the Robinson-Schensted correspondence to differential posets, Ph.D. thesis, M.I.T., Cambridge, Massachusetts, 1991.

[10] Bruce E. Sagan, The symmetric group, Wadsworth \& Brooks/Cole, Pacific Grove, California, 1987.

[11] Marcel Paul Schützenberger, Quelques remarques sur une construction de Schensted, Mathematica Scandinavica 12 (1963), 117-128. MR 0190017 (32 \#7433)

[12] Richard P. Stanley, Enumerative combinatorics. Vol. 2, Cambridge Studies in Advanced Mathematics, vol. 62, Cambridge University Press, Cambridge, 1999, With a foreword by Gian-Carlo Rota and appendix 1 by Sergey Fomin. MR 1676282 (2000k:05026)

[13] Sheila Sundaram, On the combinatorics of representations of the symplectic group, ProQuest LLC, Ann Arbor, MI, 1986, Thesis (Ph.D.)-Massachusetts Institute of Technology. MR 2941115

[14] Orthogonal tableaux and an insertion algorithm for $\mathrm{SO}(2 n+1)$, J. Combin. Theory Ser. A 53 (1990), no. 2, 239-256. MR 1041447 (91c:05199)

[15] _ , Tableaux in the representation theory of the classical Lie groups, Invariant theory and tableaux (Minneapolis, MN, 1988), IMA Vol. Math. Appl., vol. 19, Springer, New York, 1990, pp. 191-225. MR 1035496 (91e:22022)

[16] Takeshi Tokuyama, Highest weight vectors associated with some branchings, The Arcata Conference on Representations of Finite Groups (Arcata, Calif., 1986), Proc. Sympos. Pure Math., vol. 47, Amer. Math. Soc., Providence, RI, 1987, pp. 541-545. MR 933441 (89h:22029)

[17] Bruce W. Westbury, Invariant tensors and the cyclic sieving phenomenon, Preprint (2009).

Fakultät FÜr Mathematik und Geoinformation, TU Wien, Austria

E-mail address: martin.rubey@math.uni-hannover.de

Department of Mathematics, Michigan State University, East LansING, MI 48824-1027, USA

E-mail address: sagan@math.msu.edu

Department of Mathematics, University of Warwick, Coventry, CV4 $7 \mathrm{AL}$

E-mail address: Bruce.Westbury@warwick.ac.uk 